\title{
openheart Outcomes of cardiac resynchronisation therapy in patients with heart failure with atrial fibrillation: a systematic review and meta-analysis of observational studies
}

\author{
Usman Mustafa, ${ }^{\circ 1,2}$ Jessica Atkins, ${ }^{1,2,3}$ George Mina, ${ }^{1,2,3}$ Desiree Dawson, ${ }^{1,2,3}$ \\ Catherine Vanchiere, ${ }^{1,2,3}$ Narendra Duddyala, ${ }^{1,2,3}$ Ryan Jones, ${ }^{1,2,3}$ Pratap Reddy, ${ }^{1,2,3}$ \\ Paari Dominic ${ }^{1,2,3}$
}

\begin{abstract}
- Additional material is published online only. To view please visit the journal online (http://dx.doi.org/10.1136/ openhrt-2018-000937).

To cite: Mustafa U, Atkins J, Mina G, et al. Outcomes of cardiac resynchronisation therapy in patients with heart failure with atrial fibrillation: a systematic review and metaanalysis of observational studies. Open Heart 2019;6:e000937. doi:10.1136/ openhrt-2018-000937
\end{abstract}

UM and JA contributed equally.

Received 18 September 2018 Revised 22 November 2018 Accepted 20 January 2019

Check for updates

(c) Author(s) (or their employer(s)) 2019. Re-use permitted under CC BY-NC. No commercial re-use. See rights and permissions. Published by BMJ.

For numbered affiliations see end of article.

Correspondence to Dr Paari Dominic; pdomi2@ Isuhsc.edu

\section{ABSTRACT}

Background Cardiac resynchronisation therapy (CRT) is beneficial in selected patients with heart failure (HF) in normal sinus rhythm (NSR). We sought to evaluate the impact of CRT with or without atrioventricular junction (AVJ) ablation in patients with $\mathrm{HF}$ with concomitant atrial fibrillation (AF)

Methods and results Literature was searched (inception through 30 August 2017) for observational studies that reported outcomes in patients with HF with CRT and AF that reported all-cause and cardiovascular mortality. Thirty-one studies with 83,571 patients were included. CRT did not decrease mortality compared with internal cardioverter defibrillator or medical therapy alone in patients with $\mathrm{HF}$ and $\mathrm{AF}$ with indications for CRT (OR: $0.851,95 \% \mathrm{Cl} 0.616$ to $\left.1.176, \mathrm{p}=0.328, \mathrm{I}^{2}=86.954\right)$. CRT-AF patients had significantly higher all-cause and cardiovascular mortality than CRT-NSR patients ([OR: $1.472,95 \% \mathrm{Cl} 1.301$ to $1.664, \mathrm{p}=0.000$ ] and [OR: 1.857 , $95 \% \mathrm{Cl} 1.350$ to $2.554, \mathrm{p}=0.000$ ] respectively). Change in left ventricular ejection fraction was not different between CRT patients with and without $A F(p=0.705)$. AVJ ablation, however, improved all-cause mortality in CRT-AF patients when compared with CRT-AF patients without AVJ ablation (OR: $0.485,95 \% \mathrm{Cl} 0.247$ to $0.952, \mathrm{p}=0.035$ ). With AVJ ablation, there was no difference in all-cause mortality in CRT-AF patients compared with CRT-NSR patients (OR: $1.245,95 \% \mathrm{Cl} 0.914$ to $1.696, p=0.165$ ).

Conclusion The results of our meta-analysis suggest that AF was associated with decreased CRT benefits in patients with HF. CRT, however, benefits patients with AF with AVJ ablation.

\section{INTRODUCTION}

Atrial fibrillation (AF) and heart failure (HF) frequently coexist, and one condition often predisposes to the development of the other. These conditions are often synergistic and are associated with higher morbidity and mortality when they occur in the same

\section{Key messages}

What is already known about this subject?

- Currently patients with heart failure and wide QRS complex undergo cardiac resynchronisation therapy (CRT) regardless of their native rhythm.

What does this study add?

- Patients in atrial fibrillation may not benefit from a higher risk CRT implantation procedure compared with internal cardiac defibrillators, without atrioventricular junction ablation making the risk-benefit ratio suboptimal.

How might this impact on clinical practice?

- The results of this meta-analysis change the way we approach patients with heart failure with atrial fibrillation who fit criteria for a CRT device and at the least raises the urgent need for large randomised controlled trials.

patient. In addition, when $\mathrm{AF}$ and $\mathrm{HF}$ co-occur, the management of one can make the management of the other challenging. Despite advancement in pharmacologic treatment for AF, it is associated with worse outcomes in patients with HF compared with those in normal sinus rhythm (NSR). ${ }^{1}$ Cardiac resynchronisation therapy (CRT) (a class IIa indication for $\mathrm{AF}$ ) has shown to improve morbidity and mortality in patients with HF who are in NSR by improving ventricular synchrony via biventricular (Bi-V) pacing. Whether CRT similarly improves outcome in patients with $\mathrm{HF}$ in $\mathrm{AF}$ is a matter of ongoing debate. Appropriate CRT pacing is difficult to ensure in the setting of $\mathrm{AF}$, as loss of atrioventricular $(\mathrm{AV})$ synchrony and uncontrolled ventricular rate could cause suboptimal delivery of CRT pacing. ${ }^{23}$ Nevertheless, some 
studies have reported improved clinical and functional outcomes in patients with AF with CRT, ${ }^{4-11}$ while others have suggested that CRT is only beneficial in patients with $\mathrm{AF}$ after atrioventricular junction (AVJ) ablation, ${ }^{12-16}$ an intervention which makes $\mathrm{Bi}-\mathrm{V}$ pacing failure unlikely. The increasing incidence of $\mathrm{AF}$ in patients with $\mathrm{HF}$ and accumulating evidence from small and medium-sized underpowered observational studies regarding the use of CRT in patients with AF make this an appropriate time to revisit its use in this patient population.

The aims of our meta-analysis are (1) to study the impact of CRT on long-term survival and clinical outcomes in patients with $\mathrm{HF}$ and AF and (2) to investigate whether AVJ ablation adds any benefit to CRT in AF patient subgroup.

\section{METHODS}

Our systematic review and meta-analysis is in accordance with recommendations of the Meta-analysis of Observational Studies in Epidemiology group. ${ }^{17}$ The research questions, inclusion and exclusion criteria, study quality assessment tools and prespecified subgroups were established with a written protocol prior to the review and is available at http:/ / www.lsuhscardio.com/protocols/

\section{Inclusion criteria}

Observational studies reporting outcomes in CRT patients with persistent or paroxysmal AF or atrial arrhythmias were included. AF or atrial arrhythmias in these studies could be defined by the history and/or by prospective diagnostic data from the CRT devices if they were uniformly applied throughout the study groups. Studies were included if they reported incidence of all-cause mortality, cardiac mortality, change in left ventricular ejection fraction (LVEF), follow-up New York Heart Association (NYHA) status or change in 6 min walk distance (6MWD). Studies with a mean follow-up duration of at least 12 months to assess mortality or at least 6 months to assess functional improvement were included.

1. For the meta-analysis comparing outcomes in patients with $A F$ and $H F$ with CRT to patients with AF with internal cardioverter defibrillator (ICD) or optimal medical management (online supplementary figure 10-Analysis 1): Studies (retrospective and prospective) comparing outcomes in patients with $\mathrm{AF}$ and $\mathrm{HF}$ with CRT to patients with $\mathrm{AF}$ and $\mathrm{HF}$ who otherwise have indications for a CRT device but had only an ICD implanted or were only medically treated were included.

2. For the meta-analysis comparing outcomes in CRT patients with and without atrial arrhythmias (online supplementary figure 10-Analysis 2): Studies (retrospective and prospective) comparing outcomes in CRT patients who have a history of AF or atrial arrhythmia or who had AF or atrial arrhythmia during the follow-up period to CRT patients in NSR were included. We identified prespecified subgroups within this group of studies as follows: (A) studies that compared only CRT-AF patients who underwent AVJ ablation with CRT-NSR patients and/or reporting outcomes separately for this subgroup and studies that compared only CRT-AF patients who did not undergo AVJ ablation with CRTNSR patients and/or reporting outcomes separately for this subgroup; (B) studies with less than 30\% CRTAF patients who have undergone an AVJ ablation.

3. For the meta-analysis comparing outcomes in CRT patients with $A F$ with and without concomitant AVJ ablation (online supplementary figure 10-Analysis 3): Studies (retrospective and prospective) comparing outcomes in CRT patients with $\mathrm{AF}$ who have undergone AVJ ablation to CRT patients with AF managed with rate-slowing medications were included.

\section{Exclusion criteria}

Studies were excluded if they (1) lacked a control group; (2) had inadequate data on baseline characteristics; (3) were published only in abstract form; (4) were non-English studies with no English translation; (5) included patients with AF without HF who underwent AVJ ablation and CRT placement; (6) compared CRT pacing with right ventricular (RV)-only pacing in patients with AF who underwent AVJ ablation; and (7) compared only short-term left ventricular (LV) physiological parameters between AF and NSR arms.

\section{Search strategies}

MEDLINE, CINAHL, Web of Science, Cochrane Central and Google Scholar were searched (inception through 30 August 2017) independently by two investigators (JA and PD) using keywords: atrial fibrillation AND cardiac resynchronization therapy and atrioventricular junction ablation AND cardiac resynchronization therapy. The 'Related Article' feature on PubMed and a manual search of references were also used to identify additional studies. We reviewed the full text of relevant articles. English translations, if necessary, were obtained. Grey literature sources and unpublished studies were not included. Studies led by the same authors were included if they examined different populations. Titles and abstracts were independently reviewed by two authors (JA and $\mathrm{PD})$ and cross-verified by a third reviewer (UM) for inclusion. Details of the search strategy are reported in online supplementary figure 9 .

\section{Data extraction and assessment of study quality}

For each study, all data elements uniformly reported across most studies were extracted by two reviewers (JA and UM) and were cross-verified by a third reviewer (DD). The study characteristics are shown in table 1 and online supplementary tables 1-3. The quality of each study and risk of bias were evaluated with the Newcastle-Ottawa Quality Assessment Scale for non-randomised studies. ${ }^{18}$ The following characteristics were assessed for sources of bias: (1) patient selection including definitions of exposure and representation of the larger population; (2) comparability of study groups and controlling for 
confounding factors by design or analysis; and (3) assessment and documentation of outcome including duration and loss of follow-up. Studies were graded as 'poor' if they met four or less of the nine criteria, 'fair' if they met five to six criteria and 'good' if they met more than six criteria. The quality assessment of individual studies is reported alongside baseline variables in tables 1 and online supplementary tables 1 and 3 . All disagreements between reviewers were resolved by consensus.

\section{Statistical method}

For mortality, data were extracted as either OR or event rate. If $\mathrm{HR}$ was available, it was considered as the best estimate of OR. If both univariate and multivariate analyses were available, data from multivariate analyses were taken. In studies reporting outcomes separately for patients with history of $\mathrm{AF}$ and patients who developed AF during follow-up, these two were combined without overlap. ${ }^{19}$ Pooled ORs and 95\% CIs were calculated using the more conservative DerSimonian and Laird random effects model. ${ }^{20}$ Network analysis for mortality in patients with $\mathrm{AF}$ was performed using a Bayesian Markov chain MonteCarlo model. ${ }^{21}$ OR and $95 \%$ credible intervals (CrI) were calculated using random effects model with informative priors. ${ }^{22} 23$ The Bayesian analysis was performed using WinBUGS V.1.4 (MRC Biostatistics Unit, Cambridge, UK) and the Microsoft Excel-based tool (NetMetaXL). ${ }^{24}$ For LVEF and 6MWD, the final and baseline values were extracted, the difference between the two calculated and weighted mean difference (WMD) between the groups was analysed. As a majority of the studies did not report baseline NYHA class, the final NYHA class at follow-up was extracted and WMD analysed. All tests were two sided, and a $p$ value $<0.05$ was deemed significant. Sampling error within studies was assessed by examining the distribution of baseline variables between study groups. Heterogeneity was assessed by the $\mathrm{I}^{2}$ statistic, which describes the percentage of total variation across studies that is due to heterogeneity rather than chance. $\mathrm{I}^{2}>50 \%$ was considered significant heterogeneity. ${ }^{25}{ }^{26}$ Potential publication bias was assessed by visual inspection of funnel plots, in which SEs were plotted against log ORs, as well as Egger's regression intercept. All statistical analyses were performed using Comprehensive Meta-Analysis V.3 (Biostat, Englewood, NJ).

\section{RESULTS}

This meta-analysis included 31 studies $^{4-16} 19$ 27-43 with 83 571 patients. All studies were available as full text. Six studies were excluded: one due to lack of a control group ${ }^{44}$; second compared outcomes based on ventricular rates in CRT-AF patients ${ }^{3}$; third did not provide outcomes specifically for the CRT and ICD subsets of the study population with and without $\mathrm{AF}^{45}$; the fourth compared patients with ischaemic and non-ischaemic cardiomyopathy with AVJ ablation and CRT placement ${ }^{46}$; and the last two tested only short-term LV functional parameters. ${ }^{47}$ There were 30263 patients with AF, of which 25791 had CRT. Among patients with AF with CRT, 980 had undergone AVJ ablation (11\%-100\% per study). There were 53308 patients with CRT-NSR in this study.

Furthermore, two studies ${ }^{32} 37$ used the results from the Resynchronization for Ambulatory Heart Failure Trial $(\mathrm{RAFT})^{49}$; however, Wilton $e t a l^{32}$ prospectively assessed RAFT patients without AF who developed AF after randomisation, and Healey et $a l^{37}$ analysed outcome only in those RAFT patients who had pre-existing permanent AF. As they were assessing different populations, both studies were included in the analysis.

\section{Outcomes in patients with AF with and without CRT}

The study characteristics are included in online supplementary table 1 . The survival of patients with AF who received CRT was compared with patients with $\mathrm{AF}$ with indications for CRT who either received an ICD or were medically managed. Seven studies ${ }^{31} 3237-41$ with 13559 patients were included in the meta-analysis of mortality and $\mathrm{HF}$ hospitalisation in patients with $\mathrm{AF}$ with and without CRT. Four of the studies were post hoc analyses of the classic CRT trials (The Comparison of Medical Therapy, Pacing, and Defibrillation in Heart Failure [COMPANION] trial, Multicenter Automatic Defibrillator Implantation Trial with Cardiac Resynchronization Therapy [MADIT-CRT] trial, Resynchronization-Defibrillation for Ambulatory Heart Failure [RAFT] trial and The Cardiac Resynchronization-Heart Failure [CARE$\mathrm{HF}$ ] trial). One study compared CRT-Defibrillator (CRT-D) patients from the National Cardiovascular Data Registry (NCDR) with a historical cohort of patients with HF with indications for CRT treated only with medical therapy from the Acute Decompensated Heart Failure National Registry. ${ }^{40}$ The recent study by Khazanie $e t a l^{39}$ compared patients with $\mathrm{AF}$ and $\mathrm{HF}$ with indications for CRT-D, who received a CRT-D, with patients who received an ICD only in a Medicare beneficiary subset of the NCDR. Five studies compared outcomes in patients with AF with CRT with comparable patients with AF with ICD, whereas two studies ${ }^{31} 40$ compared outcomes between patients with AF with CRT and comparable patients with AF treated with optimal medical therapy. Mean follow-up was 1-3 years. Meta-analysis showed no significant benefit of CRT on mortality (figure 1A, OR: $0.851,95 \%$ CI 0.616 to $1.176, \mathrm{p}=0.328, \mathrm{I}^{2}=86.954$ ) or on composite outcome of mortality or HF hospitalisation (online supplementary figure 1, OR: $0.771,95 \%$ CI 0.550 to $1.082, \mathrm{p}=0.133$, $\left.\mathrm{I}^{2}=55.363\right)$ in patients with AF. Funnel plot analysis of CRT versus ICD in patients with AF showed no significant publication bias (online supplementary figure 2).

\section{Outcomes in CRT patients with and without AF}

Twenty-four observational studies were included for the comparison of outcomes in CRT patients with AF to CRT patients in NSR. The study characteristics are included in table 1 and online supplementary table 2 . 


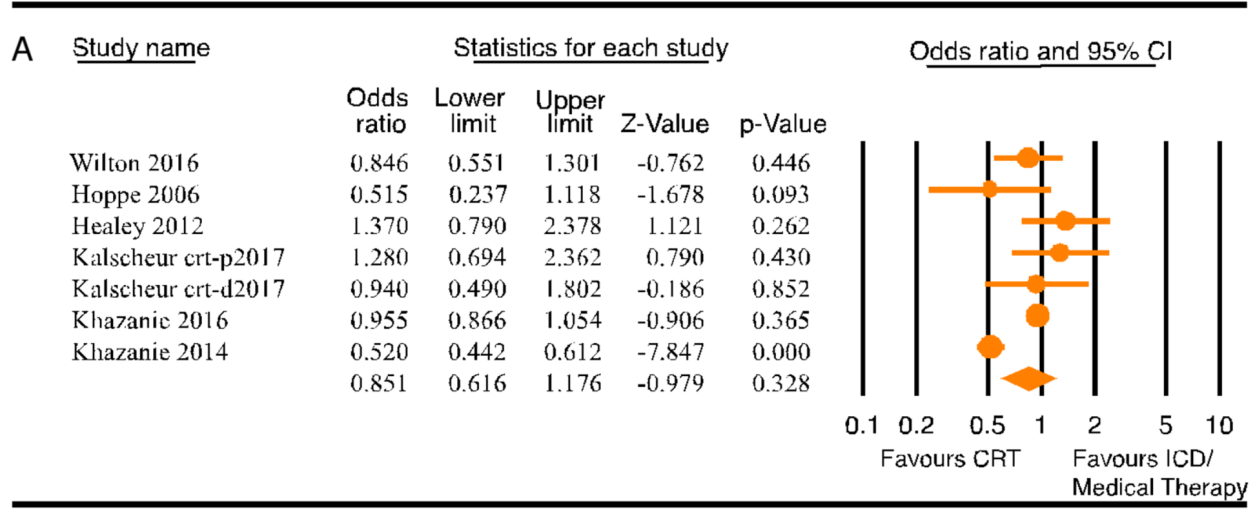

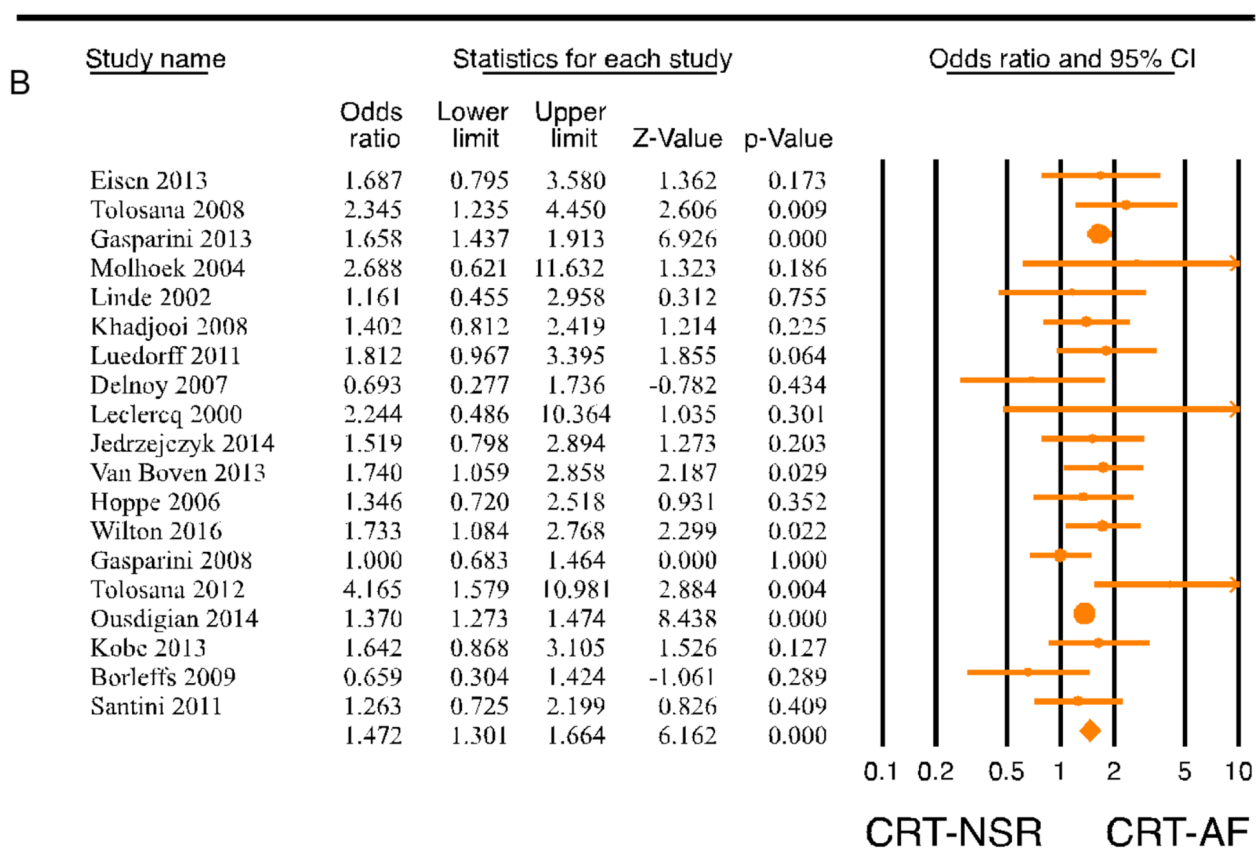

Figure 1 (A) Forest plot comparing mortality in patients with AF with CRT versus ICD/medical therapy. (B) Forest plot comparing all-cause mortality (ACM) in patients with heart failure (HF) with CRT-AF versus CRT-NSR. AF, atrial fibrillation; CRT, cardiac resynchronisation therapy; ICD, internal cardioverter defibrillator; NSR, normal sinus rhythm.

\section{Mortality}

Nineteen studies ${ }^{4-9} 121319$ 27-36 with 68782 patients were included in the meta-analysis of all-cause mortality, and 10 studies $^{4-81213153233}$ with 10628 patients were included in the meta-analysis of cardiovascular mortality. Follow-up period for the studies ranged from 1 to 7 years. All-cause and cardiovascular mortality was reported at year 1 or later in the studies, and for the purpose of this meta-analysis we extracted the longest reported mortality. All-cause mortality was significantly higher in CRT patients with AF when compared with CRT patients with NSR (figure 1B, OR: $1.472,95 \%$ CI 1.301 to $\left.1.664, \mathrm{p}=0.000, \mathrm{I}^{2}=29.956\right)$. Funnel plot of these 17 studies showed no significant publication bias (online supplementary figure 3). Cardiovascular mortality was also significantly higher in CRT-AF patients compared with CRT-NSR patients (figure 2; OR: $1.857,95 \%$ CI 1.350 to $\left.2.554, \mathrm{p}=0.000, \mathrm{I}^{2}=61.795\right)$.
Furthermore, we attempted through several sensitivity analyses to explore whether the type of AF or the timing of AF influenced the mortality outcome. As very few studies included patients with paroxysmal AF, we could not assess its effect on patients with CRT. When patients with paroxysmal AF were excluded from analysis, all-cause mortality remained significantly higher in CRT patients with permanent AF compared with NSR (OR: $1.581,95 \%$ CI 1.184 to $2.110, \mathrm{p}=0.002$, data not shown). Mortality among CRT patients with AF compared with CRT patients with NSR was unaffected by excluding patients with device-detected AF from analysis (OR: $1.506,95 \%$ CI 1.316 to $1.723, \mathrm{p}=0.000$, data not shown). Mortality analysis of four studies with device-detected AF alone showed a similar trend in increase in mortality in CRT-AF patients compared with CRT-NSR patients but was not statistically significant (OR: 1.276, 95\% CI 0.892 


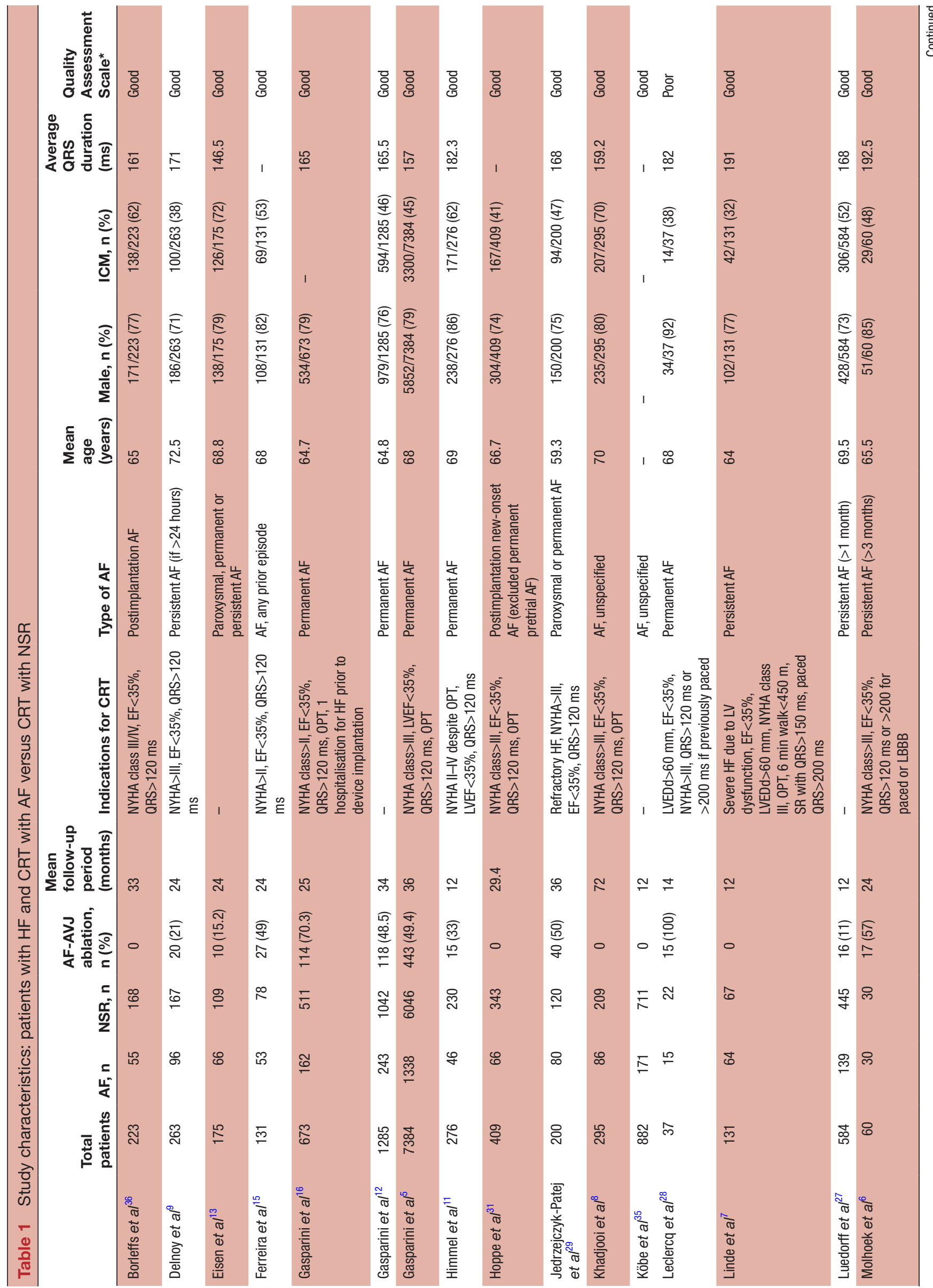




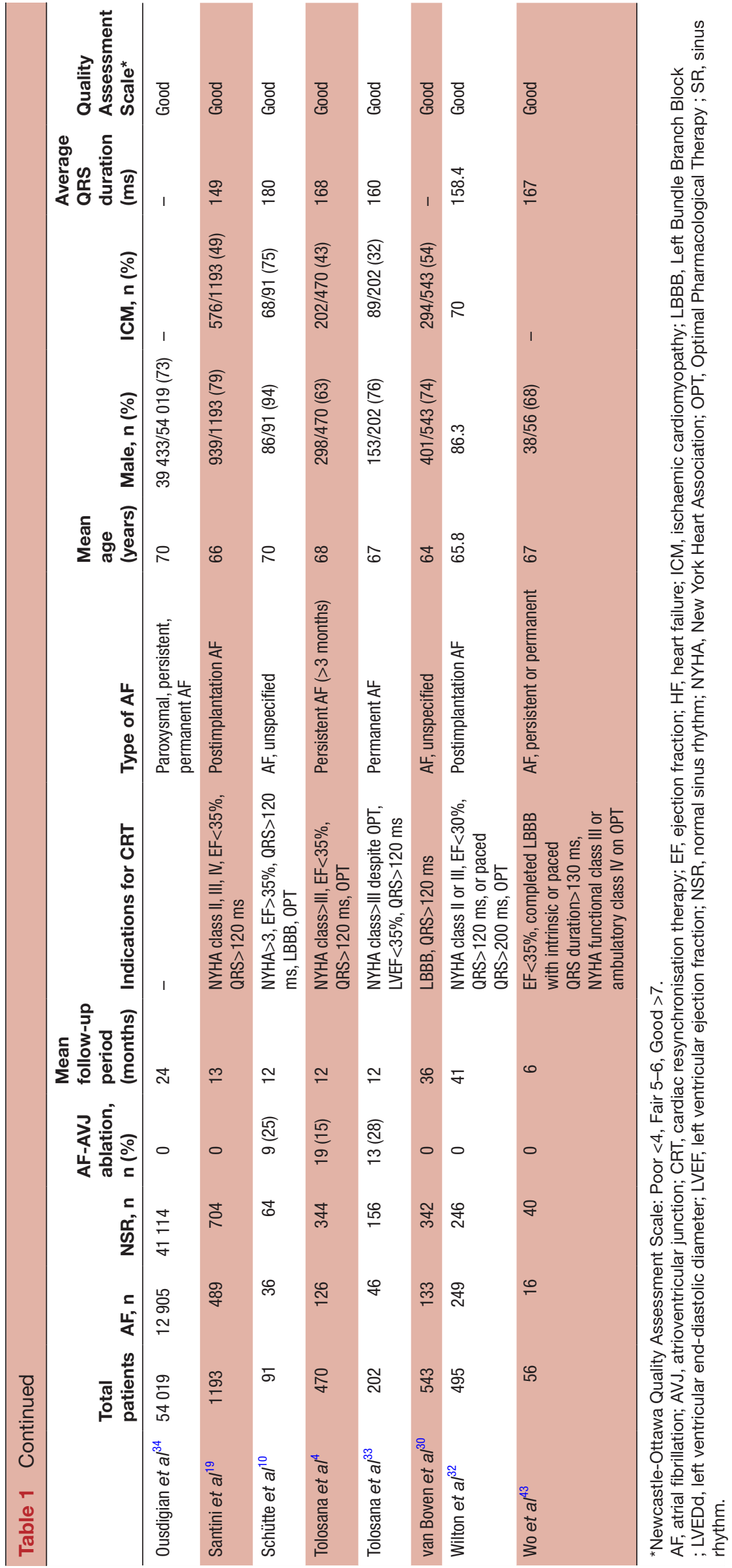

$\frac{0}{0}$

$\stackrel{1}{\mathbb{1}}$

$\stackrel{?}{\vec{*}}$

둥

$\frac{\bar{D}}{\bar{D}}$

क

$\vec{\circ}$

$\vec{\omega}$

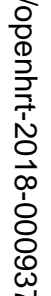


Study name

\begin{tabular}{|c|c|c|c|c|c|c|c|}
\hline & $\begin{array}{c}\text { Odds } \\
\text { ratio }\end{array}$ & $\begin{array}{l}\text { Lower } \\
\text { limit }\end{array}$ & $\begin{array}{c}\text { Upper } \\
\text { limit }\end{array}$ & Z-Value & -Value & & \\
\hline Eisen 2013 & 1.244 & 0.548 & 2.821 & 0.522 & 0.601 & & \\
\hline Tolosana 2008 & 3.746 & 1.782 & 7.873 & 3.484 & 0.000 & & \\
\hline Gasparini 2013 & 1.843 & 1.562 & 2.175 & 7.241 & 0.000 & & \\
\hline Molhoek 2004 & 2.250 & 0.507 & 9.993 & 1.066 & 0.286 & & \\
\hline Linde 2002 & 1.079 & 0.419 & 2.782 & 0.158 & 0.874 & & \\
\hline Khadjooi 2008 & 1.379 & 0.777 & 2.446 & 1.098 & 0.272 & & \\
\hline Ferreira 2008 & 11.360 & 2.389 & 54.033 & 3.054 & 0.002 & & \\
\hline Wilton 2016 & 1.588 & 0.923 & 2.733 & 1.671 & 0.095 & & \\
\hline Gasparini 2008 & 1.089 & 0.727 & 1.633 & 0.415 & 0.678 & & - \\
\hline \multirow[t]{4}{*}{ Tolosana 2012} & 6.496 & 2.234 & 18.889 & 3.436 & 0.001 & & \\
\hline & 1.857 & 1.350 & 2.554 & 3.809 & 0.000 & & \\
\hline & & & & & & 0.5 & 1 \\
\hline & & & & & & CRT-I & CRT-AF \\
\hline
\end{tabular}

Forest plot showing higher cardiovascular mortality in CRT-AF compared to CRT-NSR patients

Figure 2 Forest plot of composite endpoint in patients with AF with CRT versus ICD/GDMT. Forest plot of cardiovascular mortality in CRT patients with AF versus NSR. AF, atrial fibrillation; CRT, cardiac resynchronisation therapy; GDMT, goal directed medical therapy; ICD, internal cardioverter defibrillator; NSR, normal sinus rhythm.

to $1.825, \mathrm{p}=0.182$, data not shown). Finally, a separate meta-regression of cardioprudent drugs including betablockers and aldosterone antagonists showed no statistically significant impact on all-cause mortality $(\mathrm{p}=0.743$, $\mathrm{R}^{2}=0$ and $\mathrm{p}=0.473, \mathrm{R}^{2}=0$ respectively).

\section{Left ventricular ejection fraction}

A positive change in LVEF, as reported in 16 studies ${ }^{4-111316272831333643}$ with 11338 patients at follow-up, was surprisingly comparable in CRT patients with NSR (8.4\%, ranging from $1 \%$ to $14.9 \%$ ) and CRT patients with $\mathrm{AF}(8.6 \%$, ranging from $3 \%$ to $17 \%$, online supplementary figure 4 , standardised mean difference (SMD): $0.256,95 \%$ CI -1.072 to $\left.1.585, \mathrm{p}=0.705, \mathrm{I}^{2}=99.711\right)$.

\section{NYHA class}

Eleven studies ${ }^{6-11} 1527283136$ with 2509 patients were included in this analysis. The NYHA class at the end of follow-up was significantly higher (online supplementary figure 5, SMD: 0.604 , 95\% CI 0.195 to 1.012 , $\left.\mathrm{p}=0.004, \mathrm{I}^{2}=94.327\right)$ in CRT patients with AF $(2.1 \pm 0.65)$ when compared with CRT patients in sinus rhythm (SR) $(2.0 \pm 0.64)$, although it is unclear if this difference is clinically relevant.

\section{Six-minute walk distance}

Eight studies ${ }^{46-9} 323336$ with 2139 patients were included in this meta-analysis of change in 6MWD. The mean increment in distance travelled was $70.53 \pm 21.82 \mathrm{~m}$ in CRT patients with $\mathrm{AF}$ and was comparable in CRT patients in SR at $84.23 \pm 15.26$ (online supplementary figure 6, SMD: $-0.699,95 \%$ CI -1.828 to $0.430, \mathrm{p}=0.225, \mathrm{I}^{2}=99.128$ ).

\section{Outcomes in CRT patients with AF compared with patients in} NSR based on AVJ ablation status

To assess if AVJ ablation played any role in the mortality outcome of CRT-AF patients, CRT-AF patients from studies with less than $30 \%$ of AVJ ablation patients were compared with CRT-NSR patients (17 studies ${ }^{4} 5$ 7-9 12131927 29-36 with 68242 patients). This analysis showed significantly higher all-cause mortality in CRT-AF patients compared with CRT-NSR patients (online supplementary figure 7, OR: $1.542,95 \%$ CI 1.341 to $1.774, \mathrm{p}=0.000, \mathrm{I}^{2}=39.187$ ). Then, mortality difference among CRT-AF patients with and without AVJ ablation was compared (five randomised and non-randomised studies ${ }^{12-14} 2942$ with 1969 patients; see online supplementary table 3 for study characteristics). All-cause mortality in CRT-AF patients with AVJ ablation was significantly lower than CRT-AF patients without AVJ ablation (figure 3, OR: $0.485,95 \%$ CI 0.247 to 0.952 , $\left.\mathrm{p}=0.035, \mathrm{I}^{2}=54.294\right)$. Publication bias analysis of studies with CRT-AF patients with and without AVJ ablation was not performed due to the small number of studies. Cardiovascular mortality was not different among CRT-AF patients with and without AVJ ablation as shown by analysis of three studies (no figure included, OR: 0.764, 95\% CI 0.580 to $1.005, \mathrm{p}=0.054)$. In addition, a subgroup analysis comparing CRT-NSR patients to CRT-AF patients was performed based on whether CRT-AF patients underwent AVJ ablation. The subgroup analysis of CRT patients with and without AF based on AVJ ablation showed that CRT-AF patients without AVJ ablation (figure 4, OR: $1.492,95 \%$ CI 1.250 to $1.654, p=0.000)$ but not CRT-AF patients with AVJ ablation (figure 4, OR: 1.243, 95\% CI 0.911 to $1.696, p=0.169$ ) had significantly higher all-cause mortality compared with CRT-NSR patients ( $p$ value between groups was 0.3 ). We then performed a network analysis for all-cause mortality between patients with AF 


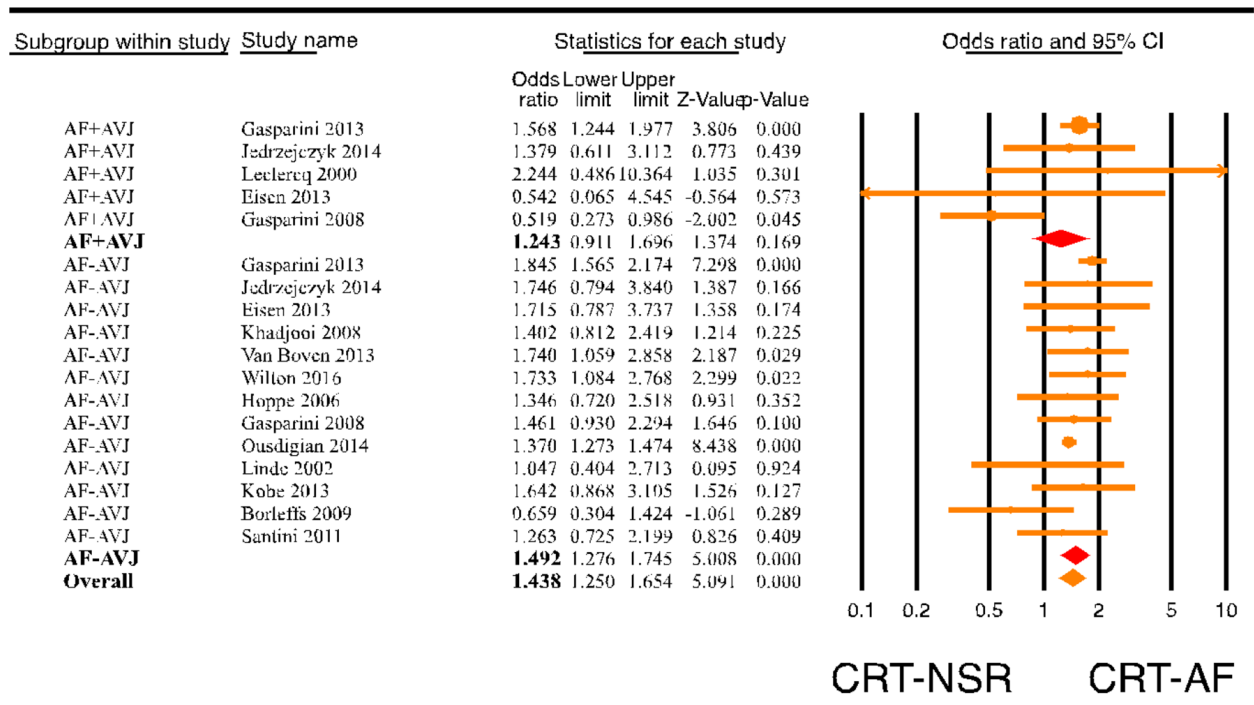

Forest plot showing odds of all cause mortality in CRT-AF compared to CRT-NSR patients based on AVJ ablation status

Figure 3 Forest plot comparing all-cause mortality (ACM) in CRT-AF versus CRT-NSR in patients based on atrioventricular junction ablation (AVJA) status. AF, atrial fibrillation; CRT, cardiac resynchronisation therapy; NSR, normal sinus rhythm.

who received CRT with AVJ ablation and patients with AF who meet CRT criteria but are on goal directed medical therapy with or without ICD. This analysis showed a non-significant trend of survival benefit from AVJ ablation in CRT patients with AF as compared with medical therapy alone (online supplementary figure 11, OR: $0.63,95 \% \mathrm{CrI} 0.35$ to 1.05 ). Finally, ejection fraction (EF) improvement in CRT-AF patients was compared with CRT-NSR patients grouped by their AVJ ablation status. CRT-AF patients who underwent AVJ ablation had a trend towards larger EF improvement (online supplementary figure 8, SDM: $4.597,95 \%$ CI -0.643 to 9.836 , $\mathrm{p}=0.086$ ) compared with the CRT-NSR patients and CRT-AF patients who did not undergo AVJ ablation (online supplementary figure 8, SDM: -1.148 , 95\% CI -5.993 to $3.696, \mathrm{p}=0.642$, $\mathrm{p}$ value between the groups was 0.11 ). Together, these results suggest that AVJ ablation does improve mortality in patients with AF getting a CRT device.

\section{DISCUSSION}

This meta-analysis studies the impact of CRT in patients with $\mathrm{AF}$ and HF. The main findings of our analysis are: (1) patients with HF with indications for CRT do not have mortality benefit from CRT compared with ICD or medical therapy alone when they have coexistent AF; (2) CRT patients with AF have higher all-cause and cardiovascular mortality than CRT patients in NSR; (3) CRT patients with $\mathrm{AF}$ have higher NYHA class at follow-up compared with CRT patients in NSR; (4) functional outcome of $6 \mathrm{MWD}$ and improvement in EF was not different between the CRT groups with and without AF;

\begin{tabular}{|c|c|c|c|c|c|c|}
\hline \multirow[t]{2}{*}{ Study name } & \multicolumn{5}{|c|}{ Statistics for each study } & Odds ratio and $95 \% \mathrm{Cl}$ \\
\hline & $\begin{array}{c}\text { Odds } \\
\text { ratio }\end{array}$ & $\begin{array}{l}\text { Lower } \\
\text { limit }\end{array}$ & $\begin{array}{l}\text { Upper } \\
\text { limit }\end{array}$ & Z-Value & $\mathrm{p}$-Value & \\
\hline Gasparini 2008 & 0.355 & 0.168 & 0.752 & -2.704 & 0.007 & \\
\hline Tolosana 2013 & 0.959 & 0.505 & 1.822 & -0.127 & 0.899 & \\
\hline Eisen 2013 & 0.277 & 0.032 & 2.371 & -1.171 & 0.241 & \\
\hline Jedrzejckyk 2014 & 0.788 & 0.302 & 2.055 & -0.488 & 0.626 & -O \\
\hline \multirow[t]{3}{*}{ Dong 2010} & 0.130 & 0.030 & 0.572 & -2.700 & 0.007 & 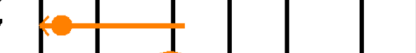 \\
\hline & 0.485 & 0.247 & 0.952 & -2.103 & 0.035 & \\
\hline & & & & & & $\begin{array}{lllllll}0.1 & 0.2 & 0.5 & 1 & 2 & 5 & 1\end{array}$ \\
\hline
\end{tabular}

Forest plot showing lower all cause mortality in AF-CRT patients who underwent AVJ ablation compared to those who did not

Figure 4 Forest plot of all-cause mortality (ACM) in CRT patient with AF comparing atrioventricular junction ablation (AVJA) versus no AVJA. AF, atrial fibrillation; CRT, cardiac resynchronisation therapy; NSR, normal sinus rhythm. 
(5) AVJ ablation tends to improve all-cause mortality in CRT patients with AF; and (6) there is no difference in all-cause mortality in CRT-AF patients who underwent an AVJ ablation versus CRT patients in NSR.

Approximately $20 \%$ of all patients with $\mathrm{HF}$ have $\mathrm{AF}^{50}$ and to our knowledge, this is the largest and most comprehensive meta-analysis to date evaluating the effectiveness of CRT in patients with AF and HF. This meta-analysis updates the information from previous meta-analyses in this subject area ${ }^{51-53}$ and adds new knowledge and perspective regarding CRT versus ICD and or optimal medical therapy in patients with AF. CRT improves morbidity and mortality and decreases HF hospitalisations in selective patients with $\mathrm{HF}$ who are in SR by improving ventricular synchrony via $\mathrm{Bi}-\mathrm{V}$ pacing. CRT devices use atrial sensing and/or pacing to time ventricular pacing in the RV and coronary sinus leads to attain effective resynchronisation. Patients with AF have no organised atrial activity and have irregular and random AV nodal conduction that can potentially prevent optimal CRT pacing. Results of our meta-analysis indicate that CRT patients with $\mathrm{AF}$ have a higher all-cause and cardiovascular mortality compared with CRT patients in SR. This could be due to one or more of the following: (1) AF may be a marker of advanced HF and worse prognosis, ${ }^{54}$ where CRT may not be as effective; (2) loss of organised atrial activity and atrial systole may lead to worse outcomes in patients with HF with or without CRT; (3) irregular intrinsic AV nodal conduction even with ventricular-sensed pacing can lead to suboptimal LV pacing and synchrony which is often underestimated in clinical practice ${ }^{55}$; and (4) patients in SR may have ventricular fusion from intrinsic conduction through the right bundle ${ }^{56}$ that might reduce the deleterious effects of RV apical pacing compared with patients with $\mathrm{AF}$ who have an AV node ablation. Our meta-analysis indicates that suboptimal $\mathrm{Bi}-\mathrm{V}$ pacing resulting in residual LV synchrony alone does not sufficiently explain increase in all-cause mortality in CRT-AF patients as there is comparable EF change in these patients in comparison to CRT-NSR patients, although this outcome may be diluted by the effects of AVJ ablation in a substantial portion of studies reporting LVEF in CRT-AF patients as evidenced by our subgroup analysis (online supplementary figure 8).

CRT is indicated in patients with AF who otherwise qualify for a CRT device if AV nodal ablation or pharmacologic rate control will ensure near $100 \%$ ventricular pacing with CRT according to the updated American College of Cardiology/American Heart Association and European Society of Cardiology guidelines. ${ }^{57}$ In clinical practice, however, rapid ventricular rate occurs in one-third of all patients with AF with CRT and is associated with suboptimal CRT pacing. ${ }^{3}$ Higher percentage of $\mathrm{Bi}-\mathrm{V}$ pacing by $\mathrm{CRT}$ is required for optimal response, which is difficult to achieve in patients with $\mathrm{AF}$, and up to two-thirds of patients with $\mathrm{AF}$ can have suboptimal CRT pacing rates leading to higher mortality. ${ }^{34}$ A meta-analysis published in 2008 showed no difference in mortality between CRT patients with and without $\mathrm{AF}^{51}$ but recent evidence incorporated in the current meta-analysis, in agreement with the study by Wilton et $a l,^{52}$ now suggests that coexisting $\mathrm{AF}$ in patients who receive CRT may be associated with increased all-cause and cardiovascular mortality, and higher NYHA class despite similar EF improvement at follow-up. While the analysis comparing mortality in CRT patients with and without $\mathrm{AF}$ shows that $\mathrm{AF}$ is associated with worse outcomes in CRT patients, it does not negate the benefits of CRT in patients with AF. To specifically investigate if CRT reduces mortality and HF hospitalisations in patients with AF, we analysed for the first time, outcomes from studies that compared CRT with ICD or medical management in patients with $\mathrm{AF}$ who otherwise were candidates for CRT. Our meta-analysis shows that patients with $\mathrm{AF}$ who received CRT had comparable death and HF hospitalisations as patients with $\mathrm{AF}$ who received ICD or medical management, suggesting that CRT perhaps does not add benefit to the patients with $\mathrm{AF}$ and $\mathrm{HF}$ with indications for CRT. In fact, significant heterogeneity in this analysis seems to be driven by the CRT versus medical management studies ${ }^{38} 40$ that skew the outcomes in favour of the CRT group as they show that CRT did reduce mortality in patients with AF, an effect that could be attributed to the defibrillator part alone.

A major limitation to optimal CRT pacing in patients with $\mathrm{AF}$ is rapid and irregular ventricular conduction through the AV node decreasing the $\mathrm{Bi}-\mathrm{V}$ pacing percentage. ${ }^{34}$ Even in patients who are aggressively rate controlled with multiple medications and trigger mode pacing, where device data show over $90 \% \mathrm{Bi}-\mathrm{V}$ pacing, more than half the patients have up to $40 \%$ of beats with fusion or pseudofusion. ${ }^{55}$ Ablation of the AVJ in patients with $\mathrm{AF}$ abolishes AV nodal conduction, allowing the ventricle to be fully paced and has been shown to lower mortality. ${ }^{59}$ Therefore, we explored whether AV junction ablation improves the outcomes of CRT in patients with AF. Among five similarly sized studies two ${ }^{12}{ }^{14}$ showed that AVJ ablation improved survival in CRT patients with AF, and three ${ }^{13942}$ showed no difference. In addition, Jedrzejczyk-Patej $e t a l^{29}$ showed that CRT-AF patients have higher all-cause mortality compared with CRT patients in SR despite AVJ ablation. Our meta-analysis shows that patients with AF with CRT have reduced all-cause mortality if they also receive concomitant $\mathrm{AV}$ junction ablation, which is consistent with the findings of a meta-analysis performed by Lopes et al. ${ }^{60}$ Furthermore, our meta-analysis of studies comparing patients with AF only with AVJ ablation or reporting outcomes separately for this subgroup compared with CRT patients in SR shows that patients with AF with CRT who undergo an AVJ ablation have similar all-cause mortality compared with CRT patients in NSR. There could be several explanations for the beneficial effects of AVJ ablation in patients with HF and AF who undergo CRT placement. First, at higher heart rates, irregular R-R intervals in patients with $\mathrm{AF}$ and $\mathrm{HF}$ impair systolic function even with $\mathrm{Bi}-\mathrm{V}$ pacing. ${ }^{61}$ Additionally, patients with $\mathrm{AF}$ and intact $\mathrm{AV}$ nodal conduction have a higher risk of inappropriate shocks which have been associated with higher all-cause mortality. ${ }^{62}$ Another possible explanation is the change in drugs or their dosage, which could cause negative inotropic effects or proarrhythmic 
effects ${ }^{63}$ or may be independently associated with higher mortality. ${ }^{64}$ Moreover, AVJ ablation has also been reported as an independent predictor of spontaneous resumption of SR in patients with AF with CRT, which can also explain favourable survival outcome in this particular subset of patients. ${ }^{65}$ While ablate and pace strategy seems very appealing, limitations of AVJ ablation including pacemaker dependency, ventricular arrhythmias ${ }^{66}$ and sudden death ${ }^{67}$ should also be considered. Presently, there are no studies comparing outcomes in patients with $\mathrm{AF}$ with indications for a CRT device who underwent AVJ ablation randomised to CRT-D or ICD alone.

Pulmonary vein isolation (PVI) is another strategy to overcome AF burden in patients with HF without making them pacemaker dependent. Although the Pulmonary Vein Isolation for Atrial Fibrillation Patients with Heart Failure trial has shown that PVI was superior to AVJ ablation and CRT pacing in patients with drug refractory AF and $\mathrm{HF}^{68}$ these patients had narrow QRS complexes and an EF less than $40 \%$. Therefore, the results of this study cannot be extrapolated to traditional patients who fulfil the criteria for CRT. Moreover, the study did not examine mortality benefit, which remains unclear with a PVI approach in this patient population.

Our present meta-analysis has important clinical implications. A substantial number of patients with HF who receive CRT may have coexistent AF, and this intervention may not alter mortality or functional outcomes in these patients, while subjecting them to the added risks of the procedure. AVJ ablation, an intervention which will ensure sufficient Bi-V capture, increasing the chances of optimal CRT response in this particular subset of patients may improve survival with CRT.

\section{Limitations}

(1) The included studies are retrospective studies or post hoc analyses of prospective randomised trials, and the endpoints were extracted as event rates; most studies did not report multivariate adjusted rate ratios or hazard ratios. (2) The studies were heterogeneous with regard to AF type and included differing patient mix of paroxysmal, persistent and permanent AF. Our sensitivity analysis of all studies with persistent or permanent AF shows that CRT-AF patients have a higher all-cause mortality compared with CRT-NSR patients. However, this analysis could not be performed for patients with paroxysmal AF as very few studies reported outcomes for this population separately. (3) The studies reported outcomes in patients with pre-existing $\mathrm{AF}$ and/ or new onset at follow-up. Our sensitivity analysis showed mortality outcomes that were similar in these two populations but the results were not statistically significant in CRT patients with device-detected AF. (4) Pacing modes in CRT devices, especially with regard to ventricular rate regulation, trigger mode or ventricular sensed response mode were not included. (5) CRT pacing percentages were not uniformly reported in all the studies. (6) Appropriate and inappropriate shocks were not uniformly reported in all the studies. (7) We did not have sufficient information regarding patients who underwent AVJ ablation in the studies comparing patients with AF (who otherwise have indications for a CRT) who received a CRT or ICD $/$ medical therapy. (8) Analysis on benefit of CRT in AF as compared with patients with $\mathrm{AF}$ who meet CRT criteria but are on medical therapy/ICD should be interpreted carefully, given heterogeneous patient population. (9) Although we found no difference in LVEF improvement between CRT patients with AF and NSR, this lack of difference might be from lack of standardised cores for measuring echocardiographic data. (10) Cardiac mortality was not uniformly specified across studies and breakdown regarding subtype (whether due to myocardial infarction [MI], decompensated HF or thromboembolic event) was only available in a limited number of studies when comparing deaths in AF and NSR. Linde $e t a l^{7}$ reported $4 \%$ of deaths related to Cerebrovascular Accident [CVA] in both the AF and NSR populations whereas $22 \%$ and $17 \%$ of deaths were related to decompensated HF in the AF and SR groups, respectively. Tolosana et $a t^{29}$ reported death due to thromboembolic disease in $2 \%$ of patients with AF compared with no deaths in NSR group, whereas decompensated $\mathrm{HF}$ was more common in $\mathrm{AF}(17.3 \%)$ than NSR $(2.5 \%)$ groups. Cardiac death, when specified, was considered due to decompensated HF, sudden death, arrhythmic death, MI or thromboembolic event.

\section{CONCLUSION}

In our meta-analysis, studies comparing CRT to ICD or medical therapy in patients with AF clearly show no additional mortality benefit with CRT. This is further supported by the evidence from studies comparing CRT outcomes in patients with AF to patients in NSR, showing higher NYHA class at follow-up, and higher cardiovascular and all-cause mortality in patients with AF with CRT despite comparable improvement in LVEF. Finally, although there are a small number of studies at this time, AVJ ablation appears to improve the outcomes of CRT in patients with AF, but further large randomised controlled trials are needed.

\section{Author affiliations}

${ }^{1}$ Department of Medicine/Division of Cardiology and Center for Cardiovascular Disease \& Sciences, Louisiana State University Health Sciences Center, Shreveport, Louisiana, USA

${ }^{2}$ Department of Medicine/Division of Cardiology and Center for Cardiovascular Disease \& Sciences, Louisiana State University Heatlh Sciences Center, Shreveport, Louisiana, USA

${ }^{3}$ Department of Medicine/Division of Cardiology and Center for Cardiovascular Disease \& Sciences, Louisiana State University Health Sciences Center Shreveport, Shreveport, Louisiana, USA

Acknowledgements We thank Betty Tucker, MSLS, for her assistance in manuscript preparation.

Contributors UM, PD, JA, DD: design. PD, JA, UM: search and reconciliation. JA, UM, DD: data extraction and cross-verification. PD, GM: data analysis and guarantor. UM, JA, CV, PD: manuscript preparation. PD, ND, RJ, PR: study supervision and expertise in field.

Funding This article was supported in part by 2 U54 GM104940-02 from the National Institute of General Medical Sciences of the National Institutes of Health which funds the Louisiana Clinical and Translational Science Center. 
Disclaimer The content is solely the responsibility of the authors and does not necessarily represent the official views of the National Institutes of Health.

Competing interests None declared.

Patient consent for publication Not required

Provenance and peer review Not commissioned; externally peer reviewed.

Data sharing statement Data are available upon reasonable request.

Open access This is an open access article distributed in accordance with the Creative Commons Attribution Non Commercial (CC BY-NC 4.0) license, which permits others to distribute, remix, adapt, build upon this work non-commercially, and license their derivative works on different terms, provided the original work is properly cited, appropriate credit is given, any changes made indicated, and the use is non-commercial. See: http://creativecommons.org/licenses/by-nc/4.0/.

\section{REFERENCES}

1. Wasywich CA, Pope AJ, Somaratne J, et al. Atrial fibrillation and the risk of death in patients with heart failure: a literature-based metaanalysis. Intern Med J 2010;40:347-56.

2. Cheng A, Landman SR, Stadler RW. Reasons for loss of cardiac resynchronization therapy pacing: insights from 32844 patients. Circ Arrhythm Electrophysiol 2012:5:884-8.

3. Boriani G, Gasparini M, Landolina M, et al. Incidence and clinical relevance of uncontrolled ventricular rate during atrial fibrillation in heart failure patients treated with cardiac resynchronization therapy. Eur J Heart Fail 2011;13:868-76.

4. Tolosana JM, Hernandez Madrid A, Brugada J, et al. Comparison of benefits and mortality in cardiac resynchronization therapy in patients with atrial fibrillation versus patients in sinus rhythm (Results of the Spanish Atrial Fibrillation and Resynchronization [SPARE] Study). Am J Cardiol 2008;102:444-9.

5. Gasparini M, Leclercq C, Lunati M, et al. Cardiac resynchronization therapy in patients with atrial fibrillation: the certify study (cardiac resynchronization therapy in atrial fibrillation patients multinational registry). JACC Heart Fail 2013;1:500-7.

6. Molhoek SG, Bax JJ, Bleeker GB, et al. Comparison of response to cardiac resynchronization therapy in patients with sinus rhythm versus chronic atrial fibrillation. Am J Cardiol 2004;94:1506-9.

7. Linde $\mathrm{C}$, Leclercq $\mathrm{C}$, Rex S, et al. Long-term benefits of biventricular pacing in congestive heart failure: results from the multisite stimulation in cardiomyopathy (MUSTIC) study. J Am Coll Cardiol 2002;40:111-8.

8. Khadjooi K, Foley PW, Chalil S, et al. Long-term effects of cardiac resynchronisation therapy in patients with atrial fibrillation. Heart 2008;94:879-83

9. Delnoy PPHM, Ottervanger JP, Luttikhuis HO, et al. Comparison of usefulness of cardiac resynchronization therapy in patients with atria fibrillation and heart failure versus patients with sinus rhythm and heart failure. Am J Cardiol 2007:99.

10. Schütte F, Lüdorff G, Grove R, et al. Atrioventricular node ablation is not a prerequisite for cardiac resynchronization therapy in patients with chronic atrial fibrillation. Cardiol J 2009;16:246-9.

11. Himmel F, Reppel M, Mortensen K, et al. A strategy to achieve crt response in permanent atrial fibrillation without obligatory atrioventricular node ablation. Pacing Clin Electrophysiol 2012;35:943-7.

12. Gasparini M, Auricchio A, Metra M, et al. Long-term survival in patients undergoing cardiac resynchronization therapy: the importance of performing atrio-ventricular junction ablation in patients with permanent atrial fibrillation. Eur Heart $J$ 2008;29:1644-52.

13. Eisen A, Nevzorov R, Goldenberg G, et al. Cardiac resynchronization therapy in patients with atrial fibrillation: a 2-year follow-up study. Pacing Clin Electrophysiol 2013;36:872-7.

14. Dong K, Shen W-K, Powell BD, et al. Atrioventricular nodal ablation predicts survival benefit in patients with atrial fibrillation receiving cardiac resynchronization therapy. Heart Rhythm 2010;7:1240-5.

15. Ferreira AM, Adragão P, Cavaco DM, et al. Benefit of cardiac resynchronization therapy in atrial fibrillation patients vs. patients in sinus rhythm: the role of atrioventricular junction ablation. Europace 2008;10:809-15.

16. Gasparini M, Auricchio A, Regoli F, et al. Four-year efficacy of cardiac resynchronization therapy on exercise tolerance and disease progression. J Am Coll Cardiol 2006;48:734-43.

17. Stroup DF, Berlin JA, Morton SC, et al. Meta-analysis of observational studies in epidemiology. A proposal for reporting. JAMA 2008;2000.

18. Wells W, Shea S. The Newcastle-Ottawa scale (NOS) for assessing the quality of nonrandomized studies in meta- analysis. Eur $J$ Epidemiol 2010;25:1-21.
19. Santini M, Gasparini M, Landolina M, et al. Device-detected atria tachyarrhythmias predict adverse outcome in real-world patients with implantable biventricular defibrillators. J Am Coll Cardiol 2011:57:167-72

20. DerSimonian R, Laird N. Meta-analysis in clinical trials. Control Clin Trials 1986;7:177-88.

21. Caldwell DM, Ades AE, Higgins JPT. Simultaneous comparison of multiple treatments: combining direct and indirect evidence. BMJ 2005;331:897-900.

22. Turner RM, Davey J, Clarke MJ, et al. Predicting the extent of heterogeneity in meta-analysis, using empirical data from the Cochrane database of systematic reviews. Int J Epidemiol 2012;41:818-27

23. Turner RM, Jackson D, Wei Y, et al. Predictive distributions for between-study heterogeneity and simple methods for their application in Bayesian meta-analysis. Stat Med 2015;34:984-98.

24. Brown S, Hutton B, Clifford T, et al. A Microsoft-Excel-based too for running and critically appraising network meta-analyses--an overview and application of NetMetaXL. Syst Rev 2014;3:110.

25. Higgins JPT, Thompson SG. Quantifying heterogeneity in a metaanalysis. Stat Med 2002;21:1539-58.

26. Higgins JPT, Thompson SG, Deeks JJ, et al. Measuring inconsistency in meta-analyses. BMJ 2003;327:557-60.

27. Luedorff G, Grove R, Kowalski M, et al. Chronisches Vorhofflimmern bei schwerer Herzinsuffizienz und CRT-Indikation. Herzschr Elektrophys 2011;22:226-32.

28. Leclercq C, Victor F, Alonso C, et al. Comparative effects of permanent biventricular pacing for refractory heart failure in patients with stable sinus rhythm or chronic atrial fibrillation. Am J Cardiol 2000;85:A9:1154-6.

29. Jędrzejczyk-Patej E, Lenarczyk R, Pruszkowska P, et al. Long-term outcomes of cardiac resynchronization therapy are worse in patients who require atrioventricular junction ablation for atrial fibrillation than in those with sinus rhythm. Cardiol J 2014;21:309-15.

30. van Boven $\mathrm{N}$, Theuns $\mathrm{D}$, Bogaard $\mathrm{K}$, et al. Atrial fibrillation in cardiac resynchronization therapy with a defibrillator: a risk factor for mortality, appropriate and inappropriate shocks. J Cardiovasc Electrophysiol 2013;24.

31. Hoppe UC, Casares JM, Eiskjaer H, et al. Effect of cardiac resynchronization on the incidence of atrial fibrillation in patients with severe heart failure. Circulation 2006;114:18-25.

32. Wilton SB, Exner DV, Wyse DG, et al. Frequency and outcomes of Postrandomization atrial tachyarrhythmias in the Resynchronization/ Defibrillation in ambulatory heart failure trial. Circ Arrhythm Electrophysiol 2016;9:e003807

33. Tolosana JM, Arnau AM, Madrid AH, et al. Cardiac resynchronization therapy in patients with permanent atrial fibrillation. Is it mandatory to ablate the atrioventricular junction to obtain a good response? Eur $J$ Heart Fail 2012;14:635-41.

34. Ousdigian KT, Borek PP, Koehler JL, et al. The epidemic of inadequate biventricular pacing in patients with persistent or permanent atrial fibrillation and its association with mortality. Circ Arrhythm Electrophysiol 2014;7:370-6.

35. Köbe J, Wasmer K, Andresen D, et al. Impact of atrial fibrillation on early complications and one year-survival after cardioverter Defibrillator implantation: results from the German device registry. Int J Cardiol 2013:168:4184-90.

36. Borleffs CJW, Ypenburg C, van Bommel RJ, et al. Clinical importance of new-onset atrial fibrillation after cardiac resynchronization therapy. Heart Rhythm 2009;6:305-10.

37. Healey JS, Hohnloser SH, Exner DV, et al. Cardiac resynchronization therapy in patients with permanent atrial fibrillation: results from the resynchronization for ambulatory heart failure trial (raft). Circ Heart Fail 2012;5:566-70.

38. Kalscheur MM, Saxon LA, Lee BK, et al. Outcomes of cardiac resynchronization therapy in patients with intermittent atrial fibrillation or atrial flutter in the companion trial. Heart Rhythm 2017:14:858-65

39. Khazanie P, Greiner MA, Al-Khatib SM, et al. Comparative effectiveness of cardiac resynchronization therapy among patients with heart failure and atrial FibrillationCLINICAL perspective. Circulation 2016;9:e002324-19.

40. Khazanie P, Hammill BG, Qualls LG, et al. Clinical effectiveness of cardiac resynchronization therapy versus medical therapy alone among patients with heart failure: analysis of the ICD registry and adhere. Circ Heart Fail 2014;7:926-34.

41. Ruwald A-C, Pietrasik G, Goldenberg I, et al. The Effect of Intermittent Atrial Tachyarrhythmia on Heart Failure or Death in Cardiac Resynchronization Therapy With Defibrillator Versus Implantable Cardioverter-Defibrillator Patients. J Am Coll Cardio 2014:63:1190-7. 
42. Tolosana JM, Trucco E, Khatib M, et al. Complete atrioventricular block does not reduce long-term mortality in patients with permanent atrial fibrillation treated with cardiac resynchronization therapy. Eur J Heart Fail 2013;15:1412-8.

43. H-T W, Chang P-C, Chen T-H, et al. Cardiac resynchronization therapy in patients with and without atrial fibrillation. Acta Cardiol Sin 2011;27:46-51.

44. Saxon LA, Crandall BG, Nydegger CC, et al. Results of the Multicenter CONTAK renewal 3 AVT clinical study of cardiac resynchronization defibrillator therapy in patients with atrial fibrillation. Heart Rhythm 2005;2.

45. Brenyo A, Link MS, Barsheshet A, et al. Cardiac resynchronization therapy reduces left atrial volume and the risk of atrial tachyarrhythmias in MADIT-CRT (Multicenter Automatic Defibrillator Implantation trial with cardiac resynchronization therapy). J Am Coll Cardiol 2011;58:1682-9.

46. Sohinki D, Ho J, Srinivasan N, et al. Outcomes after atrioventricular node ablation and biventricular pacing in patients with refractory atrial fibrillation and heart failure: a comparison between non-ischaemic and ischaemic cardiomyopathy. Europace 2014;16:880-6.

47. Buck S, Rienstra M, Maass $A H$, et al. Cardiac resynchronization therapy in patients with heart failure and atrial fibrillation: importance of new-onset atrial fibrillation and total atrial conduction time. Europace 2008;10:558-65.

48. Hay I, Melenovsky V, Fetics BJ, et al. Short-term effects of right-left heart sequential cardiac resynchronization in patients with heart failure, chronic atrial fibrillation, and atrioventricular nodal block. Circulation 2004;110:3404-10.

49. Tang ASL, Wells GA, Talajic M, et al. Cardiac-resynchronization therapy for mild-to-moderate heart failure. $N$ Engl J Med 2010;363:2385-95.

50. Anter E, Jessup M, Callans DJ. Atrial fibrillation and heart failure: treatment considerations for a dual epidemic. Circulation 2009;119:2516-25.

51. Upadhyay GA, Choudhry NK, Auricchio A, et al. Cardiac resynchronization in patients with atrial fibrillation. J Am Coll Cardiol 2008;52:1239-46.

52. Wilton SB, Leung AA, Ghali WA, et al. Outcomes of cardiac resynchronization therapy in patients with versus those without atria fibrillation: a systematic review and meta-analysis. Heart Rhythm 2011;8:1088-94

53. Yin J, Hu H, Wang Y, et al. Effects of atrioventricular nodal ablation on permanent atrial fibrillation patients with cardiac resynchronization therapy: a systematic review and meta-analysis. Clin Cardiol 2014;37:707-15.

54. Mamas MA, Caldwell JC, Chacko S, et al. A meta-analysis of the prognostic significance of atrial fibrillation in chronic heart failure. Eur $J$ Heart Fail 2009;11:676-83.

55. Kamath GS, Cotiga D, Koneru JN, et al. The utility of 12-lead Holter monitoring in patients with permanent atrial fibrillation for the identification of nonresponders after cardiac resynchronization therapy. J Am Coll Cardiol 2009;53:1050-5.

56. van Gelder BM, Bracke FA, Meijer A, et al. The hemodynamic effect of intrinsic conduction during left ventricular pacing as compared to biventricular pacing. J Am Coll Cardiol 2005;46:2305-10.

57. Tracy CM, Epstein AE, Darbar D, et al. 2012 ACCF/AHA/HRS focused update of the 2008 guidelines for Device-Based therapy of cardiac rhythm abnormalities. J Am Coll Cardiol 2012;60:1297-313.

58. Brignole M, Auricchio A, Baron-Esquivias G, et al. 2013 ESC guidelines on cardiac pacing and cardiac resynchronization therapy: the task Force on cardiac pacing and resynchronization therapy of the European Society of cardiology (ESC). developed in collaboration with the European heart rhythm Association (EHRA). Eur Heart $J$ 2013;34:2281-329.

59. Garcia B, Clementy N, Benhenda N, et al. Mortality after atrioventricular nodal radiofrequency catheter ablation with permanent ventricular pacing in atrial fibrillation: outcomes from a controlled nonrandomized study. Circ Arrhythm Electrophysiol 2016;9:e003993.

60. Lopes C, Pereira T, Barra S. Cardiac resynchronization therapy in patients with atrial fibrillation: a meta-analysis. Rev Port Cardiol 2014;33:717-25.

61. Melenovsky V, Hay I, Fetics BJ, et al. Functional impact of rate irregularity in patients with heart failure and atrial fibrillation receiving cardiac resynchronization therapy. Eur Heart $J$ 2005;26:705-11.

62. Proietti R, Labos $\mathrm{C}$, Davis $\mathrm{M}$, et al. A systematic review and meta-analysis of the association between implantable cardioverterdefibrillator shocks and long-term mortality. Can J Cardiol 2015;31:270-7.

63. Mina GS, Acharya M, Shepherd T, et al. Digoxin is associated with increased shock events and electrical Storms in patients with implantable cardioverter defibrillators. J Cardiovasc Pharmacol Ther 2018;23:142-8.

64. Chamaria S, Desai AM, Reddy PC, et al. Digoxin use to control ventricular rate in patients with atrial fibrillation and heart failure is not associated with increased mortality. Cardiol Res Pract 2015;2015:1-10.

65. Gasparini M, Steinberg JS, Arshad A, et al. Resumption of sinus rhythm in patients with heart failure and permanent atrial fibrillation undergoing cardiac resynchronization therapy: a longitudinal observational study. Eur Heart J 2010;31:976-83.

66. Nowinski K, Gadler F, Jensen-Urstad M, et al. Transient proarrhythmic state following atrioventricular junction radiofrequency ablation: pathophysiologic mechanisms and recommendations for management. Am J Med 2002;113:596-602.

67. Ozcan C, Jahangir A, Friedman PA, et al. Sudden death after radiofrequency ablation of the atrioventricular node in patients with atrial fibrillation. J Am Coll Cardiol 2002;40:105-10.

68. Khan MN, Jaïs $\mathrm{P}$, Cummings $\mathrm{J}$, et al. Pulmonary-vein isolation for atrial fibrillation in patients with heart failure. $N$ Engl J Med 2008;359:1778-85 